\title{
不同培养温度下严重侵蚀红壤的有机碳矿化特征
}

\author{
邱䂀 1,2 吕茂奎 ${ }^{1,2}$ 黄锦学 ${ }^{1,2}$ 李 伟 1,2 赵本嘉 1,2 张 浩 1,2 王恩熙 1,2 \\ 谢锦升 ${ }^{1,2,3 *}$
}

${ }^{1}$ 福建师范大学地理科学学院, 福州 350007; 湿润亚热带山地生态国家重点实验室培育基地, 福州 350007 ; $^{3}$ 福建师范大学地理研究所, 福州 350007

摘 要 研究侵蚀土壤有机质矿化及其温度敏感性 $\left(Q_{10}\right)$ 对深入认识水土流失地区土壤有机碳动态变化具有重要意义。该文 以福建省长汀县河田镇严重侵蚀区的裸露红壤为研究对象, 通过测定不同培养温度 $\left(10{ }^{\circ} \mathrm{C} 、 20{ }^{\circ} \mathrm{C}\right.$ 和 $\left.30{ }^{\circ} \mathrm{C}\right)$ 下的土壤有机碳矿 化速率、培养过程中微生物生物量碳( $\mathrm{MBC}$ )和可溶性有机碳(DOC)含量的变化, 探讨了温度对严重侵蚀红壤有机碳矿化特征 的影响及其 $Q_{10}$ 。结果表明: 温度对严重侵蚀红壤有机碳矿化具有显著影响, 温度越高土壤有机碳矿化速率和矿化率越高; 培 养过程中土壤有机碳累积矿化量与 $\mathrm{MBC}$ 显著正相关, 与 $\mathrm{DOC}$ 极显著负相关, 说明微生物生物量和可利用碳含量显著影响土 壤有机碳的矿化。尽管严重侵蚀红壤有机碳含量仅为 $1.54 \mathrm{~g} \cdot \mathrm{kg}^{-1}$, 但培养 180 天的土壤有机碳的累积矿化率高达 $22.2 \%-33.3 \%$, 表明侵蚀红壤有机碳容易被矿化。严重侵蚀红壤在 $10-20{ }^{\circ} \mathrm{C}$ 时的 $Q_{10}$ 值为 $1.41,20-30{ }^{\circ} \mathrm{C}$ 时 $Q_{10}$ 值下降到 1.06 , 土壤有机碳质量 低是导致 $Q_{10}$ 值较低的重要原因, 而严重侵蚀区的红壤长期裸露使微生物对高温产生适应性是高温时 $Q_{10}$ 值接近 1 的重要原因。 因此，在未来气候变暖的趋势下，恢复植被覆盖对减少严重侵蚀红壤有机碳矿化损失具有重要意义。

关键词 累积矿化量; 温度敏感性; 土壤有机碳; 微生物熵; 代谢熵

引用格式: 邱曦, 吕茂奎, 黄锦学, 李伟, 赵本嘉, 张浩, 王恩熙, 谢锦升 (2016). 不同培养温度下严重侵蚀红壤的有机碳矿化特征. 植物生态学报, 40, 236-245. doi: 10.17521/cjpe.2015.0364

\section{Characteristics of soil organic carbon mineralization at different temperatures in severely eroded red soil}

Qiu Xi ${ }^{1,2}$, LÜ Mao-Kui ${ }^{1,2}$, HUANG Jin-Xue ${ }^{1,2}$, LI Wei ${ }^{1,2}$, ZHAO Ben-Jia ${ }^{1,2}$, ZHANG Hao ${ }^{1,2}$, WANG En-Xi ${ }^{1,2}$, and XIE Jin-Sheng ${ }^{1,2,3^{*}}$

${ }^{1}$ School of Geographical Sciences, Fujian Normal University, Fuzhou 350007, China; ${ }^{2}$ State Key Laboratory Breeding Base of Humid Subtropical Mountain Ecology, Fuzhou 350007, China; and Institute of Geography, Fujian Normal University, Fuzhou 350007, China

\section{Abstract}

Aims It is crucial to study the soil organic matter decomposition and its temperature sensitivity for in-depth understanding of soil organic carbon (SOC) dynamic changes in severely eroded red soil.

Methods We selected the severely eroded red soil on the bare land from Hetian town of Changting County as the research object. To study the influences of temperature on SOC mineralization and their temperature sensitivity $\left(Q_{10}\right)$, the SOC mineralization rate, the associated soil microbial biomass carbon (MBC), and dissolved organic carbon (DOC) under different incubation temperatures $\left(10,20\right.$ and $\left.30^{\circ} \mathrm{C}\right)$ were measured.

Important findings The results showed that the incubation temperature significantly influenced the SOC mineralization. The mineralization ratio and the cumulative SOC mineralization rate increased with temperature. During the incubation period, the cumulative SOC mineralization was positively and significantly correlated with the soil $\mathrm{MBC}$, and negatively correlated with soil DOC, indicating that soil microbes and $\mathrm{C}$ availability can significantly influence the SOC mineralization. During the 180 days incubation period, the accumulation mineralization rate of the severely eroded red soil was as high as $22.2 \%-33.3 \%$ under the conditions of the SOC content being only 1.54 $\mathrm{g} \cdot \mathrm{kg}^{-1}$, which indicates that the eroded red soil is easily mineralized. For the severely eroded red soil, its SOC temperature sensitivity $\left(Q_{10}\right)$ value was 1.41 at low temperatures $\left(10-20{ }^{\circ} \mathrm{C}\right)$, but decreased to 1.06 at high temperatures $\left(20-30{ }^{\circ} \mathrm{C}\right)$. The relatively low $Q_{10}$ value at low temperatures was partly attributed to the low soil SOC quality. Furthermore, the long-time exposure to sunshine for the severely eroded red soil in the summer leads to the soil microbial adaptability to high temperature. As a result, its $Q_{10}$ value is close to 1 at high temperatures.

收稿日期Received: 2015-10-13 接受日期Accepted: 2016-01-18

* 通信作者Author for correspondence (E-mail: jshxie@163.com) 
This study implies that it is of great significance to restore vegetation to minimize loss SOC caused by mineralization of the eroded red soil under climate changes in the future.

Key words cumulative $\mathrm{C}$ mineralization; temperature sensitivity; soil organic carbon (SOC); microbial quotients; soil metabolic quotients

Citation: Qiu X, Lü MK, Huang JX, Li W, Zhao BJ, Zhang H, Wang EX, Xie JS (2016). Characteristics of soil organic carbon mineralization at different temperatures in severely eroded red soil. Chinese Journal of Plant Ecology, 40, 236-245. doi: $10.17521 /$ cjpe. 2015.0364

土壤碳库约占陆地生态系统碳(C)储量的 $67 \%$, 是陆地生态系统中最大的碳库(约1 $500 \mathrm{Pg} \mathrm{C}$ ) (Jenkinson et al., 1991; Hashimoto et al., 2004; Lal, 2004)。土壤碳矿化是陆地生态系统中碳循环的重要 过程, 对维持土壤碳库动态平衡起着重要作用(刘 绍辉和方精云, 1997)。温度作为土壤碳矿化的关键 调控因子备受关注。根据联合国政府间气候变化专 门委员会(IPCC)第五次报告, 从1880年到2012年, 地表和海平面的平均温度上升了 $0.85{ }^{\circ} \mathrm{C}$ (IPCC, 2013)。由于温室效应, 即使人们以后不再排放温室 气体, 地表温度也将持续上升。因此, 温度升高如何 影响土壤碳矿化已成为全球变化和碳循环研究的焦 点问题, 目前国内外有关森林、草地和水田的土壤 碳矿化及其温度敏感性的报道(艾丽等, 2007; 王清 奎等, 2007; Chatterjee et al., 2008; 王若梦等, 2013) 非常多。

我国是全球水土流失最严重的国家，水土流失 总面积为 484.74 万 $\mathrm{km}^{2}$, 占国土总面积的 $51.1 \%$ (李 智广, 2009)。福建长汀县是我国南方花岗岩地区最 典型的水土流失区, 从 20 世纪 80 年代起, 各级主管 部门相继采取许多治理措施, 水土流失得到很好的 控制, 大面积以马尾松(Pinus massoniana)为主的植 被得到不同程度的恢复(谢锦升等, 2008)。然而, 仍 存在大面积的林下裸露地, 造成严重的林下水土流 失(何圣嘉等, 2011), 而且在阳光直射下土壤温度显 著提高。因此, 研究温度如何影响裸露地土壤有机 碳的矿化过程及其温度敏感性, 对深入了解严重侵 蚀红壤有机碳动态过程及其如何响应气候变暖具有 重要意义。

本研究以福建省西部汀江上游的长汀县河田镇 未经治理的严重侵蚀红壤为研究对象, 测定了土壤 在不同培养温度 $\left(10{ }^{\circ} \mathrm{C} 、 20{ }^{\circ} \mathrm{C}\right.$ 和 $\left.30{ }^{\circ} \mathrm{C}\right)$ 下的有机碳矿 化速率和温度敏感性 $\left(Q_{10}\right)$, 并观测了不同培养阶段 土壤的可溶性有机碳(DOC) 和微生物生物量碳 $(\mathrm{MBC})$, 以及微生物代谢熵的变化特征, 旨在阐明 温度对严重侵蚀红壤有机碳矿化的影响程度及其控
制因子, 为揭示严重侵蚀红壤有机碳动态过程提供 科学依据。

\section{1 实验材料和方法}

\section{1 研究地区概况}

试验地位于福建省长汀县河田镇 $\left(25.55^{\circ}-\right.$ $25.80^{\circ} \mathrm{N}, 116.30^{\circ}-116.52^{\circ} \mathrm{E}$ ), 海拔300-500 $\mathrm{m}$ 。该地 区属中亚热带季风气候区, 年平均气温 17.5-18.8 ${ }^{\circ} \mathrm{C}$, 年降水量 $1700 \mathrm{~mm}$, 年蒸发量 $1403 \mathrm{~mm}$ 。平均 日照时间 $1924.6 \mathrm{~h}$, 平均无霜期 260 天, $\geqslant 10{ }^{\circ} \mathrm{C}$ 积 温4 100-4 $650{ }^{\circ} \mathrm{C}$ 。地带性植被为常绿榈叶林, 但已 基本被破坏殆尽, 现有植被主要以人工种植的马尾 松和次生林为主, 树种结构单一, 生长较差。该研究 区土壤为中粗粒花岗岩发育的红壤, 抗侵蚀能力弱, 属强度土壤侵蚀区, 土壤 $\mathrm{A}$ 层流失殆尽, $\mathrm{B}$ 层出露, 立地条件极差。2003年土壤侵蚀遥感资料显示, 河 田镇水土流失面积为 $13586.83 \mathrm{hm}^{2}$, 占全镇总土地 面积的 $46.68 \%$ 。

\section{2 土样采集及处理}

2014年5月上旬，选取立地条件基本一致的未 治理马尾松“小老头林”地，设置 3 个 $20 \mathrm{~m} \times 20 \mathrm{~m}$ 的 标准样方进行调查。马尾松密度 1225 株. $\mathrm{hm}^{-2}$, 平均 树高 $1.91 \mathrm{~m}$, 平均胸径 $2.8 \mathrm{~cm}$, 林下植被主要为芒萁 (Dicranopteris pedata), 盖度仅 $10 \%$ 左右。按“S”随机 采集裸地表层0-10 $\mathrm{cm}$ 土壤, 以12点组成一个土样, 重复3次。将采集的土样除去植物残体和石砾, 每个 样地土样分别混匀分成两份, 一份土样过 $2 \mathrm{~mm}$ 篎 后置于 $4{ }^{\circ} \mathrm{C}$ 冰箱保鲜备用。另一份土样一部分用于 测定土壤质量含水量, 另一部分风干后过 $0.149 \mathrm{~mm}$ 篮，用于测定土壤总有机碳、氮。采样的同时，采用 环刀法测定土壤饱和持水量(鲁如坤, 1999)。

\section{3 室内培养与土壤性质测定}

根据测定的土壤含水量, 称取相当于 $50 \mathrm{~g}$ 干土 质量的鲜土装入 $500 \mathrm{~mL}$ 特制的培养瓶中, 并调节土 壤含水量至饱和持水量的 $60 \%$, 之后用扎孔的保鲜 膜封住(既可保持通风又可防止土壤水分损失过快), 
在 $20{ }^{\circ} \mathrm{C}$ 恒温箱内预培养两周, 使土壤内部环境趋于 稳定。预培养结束后, 将土壤样品分别置于 $10{ }^{\circ} \mathrm{C}$ 、 $20{ }^{\circ} \mathrm{C} 、 30{ }^{\circ} \mathrm{C}$ 恒温培养箱中。每个温度梯度设置 23 个培养瓶，其中 5 个用于长期测定土壤碳矿化速率, 18 个用于定期破坏性取样。培养期间每 5 天采用称重 法调节土壤含水量至 $60 \%$ 饱和持水量, 同样用扎孔 保鲜膜封住。在培养第7、14、21、30、60、90、120、 150 和 180 天抽气取样, 测定 $\mathrm{CO}_{2}$ 浓度和计算矿化速 率。每次测定时, 先用标准压缩空气通入培养瓶中 洗气 $30 \mathrm{~s}$, 使培养瓶内的气体稳定后用瓶盖密封, 用注射器从培养瓶橡胶口抽取 $10 \mathrm{~mL}$ 气体, 注入密 闭气袋, 然后将培养瓶放回培养箱, $2 \mathrm{~h}$ 后采用同样 的方法取气，用气相色谱-质谱联用仪(GC-2014, Shimadzu, Kyoto, Japan)测定土壤培养第 $0 、 2 \mathrm{~h}$ 的 $\mathrm{CO}_{2}$ 浓度, 并计算出 $\mathrm{CO}_{2}$ 排放速率和 $\mathrm{CO}_{2}$ 累积排放量。在 培养的第30、90、180天每次随机取出6个破坏性取 样的土壤样品, 用于测定DOC和MBC等指标。

土壤有机碳、全氮含量用碳氮元素分析仪(Vario MAX, Elementar Analysensysteme GmbH, Hanau, Germany)测定。测定土壤 $\mathrm{pH}$ 值时, 风干土水比为 $1: 2.5$ 。土壤机械组成用土壤粒径分析系统 (SEDIMAT 4-12, UGT, Berlin, Germany)测定。浸提 得到的DOC及氯仿-熏蒸浸提法得到的 $\mathrm{MBC}$ 用总有 机碳分析仪(TOC-VCPH, Shimadzu, Kyoto, Japan)测 定。土壤微生物熵为微生物生物量碳含量与土壤有 机碳的比值(用百分比表示)。代谢商为呼吸速率与 微生物生物量碳含量的比值(单位为 $\mathrm{h}^{-1}$ )。

\section{4 数据计算与处理方法}

\subsection{1 土壤矿化速率计算}

$$
F=k \times \frac{V}{m} \times \frac{\Delta c}{\Delta t} \times \frac{273}{273+T}
$$

式中: $F$ 为气体排放速率 $\left(\mathrm{mg} \cdot \mathrm{kg}^{-1} \cdot \mathrm{h}^{-1}\right) ; k$ 是气体转化 为标准单位的系数 $\left(\mathrm{kg} \cdot \mathrm{m}^{-3}\right) ; V$ 为培养瓶内总气体体 积 $\left(\mathrm{m}^{3}\right) ; m$ 为干土质量 $(\mathrm{kg}) ; \Delta c$ 为 $\mathrm{CO}_{2}$ 浓度差 $\left(\mu \mathrm{mol} \cdot \mathrm{mol}^{-1} ; \Delta t\right.$ 为间隔时间 $(\mathrm{h}) ; T$ 为培养温度 $\left({ }^{\circ} \mathrm{C}\right)($ 李 杰等, 2014)。

\subsection{2 土壤碳矿化 $Q_{10}$ 值计算}

本研究采用Conant等(2008)采用的 $Q_{10}$ 值计算方 法, 计算公式为:

$$
Q_{10}=\left(\frac{t_{c}}{t_{w}}\right)^{\left(\frac{10}{T_{w}-T_{c}}\right)}
$$

式中 $t_{c}$ 和 $t_{w}$ 分别是当土壤有机碳的矿化率相同时, 较低培养温度 $\left(T_{c}\right)$ 和相对更高培养温度 $\left(T_{w}\right)$ 持续的 时间。用这种计算方法获得的 $Q_{10}$ 值避免了底物供给 差异造成的低估(Luo et al., 2001)。

\subsection{3 数据处理方法}

采用方差分析方法比较不同温度培养下的矿化 速率、累积矿化量、 $Q_{10}$ 、活性碳含量、微生物熵和 代谢商等指标的差异, 观测值如果出现高于平均值 2倍标准差的数据，在分析过程中将被剔除; 采用 相关分析方法分析土壤有机碳累积矿化量与MBC、 DOC含量的关系。所有数据均用Excel 2013进行处 理, 用SPSS 19.0进行差异显著性检验和相关分析。 采用Origin 9.0画图软件绘制图形。

\section{2 结果和分析}

\section{1 严重侵蚀红壤有机碳累积矿化量及其 $Q_{10}$}

严重侵蚀红壤在不同温度 $\left(10 、 20 、 30{ }^{\circ} \mathrm{C}\right)$ 下，培 养前30天矿化速率较快, 之后矿化速率相对变慢 (图1)。经过180天培养, 10、20和 $30{ }^{\circ} \mathrm{C}$ 有机碳累积矿 化量的平均值分别为 $341.21 、 468.53 、 541.19 \mathrm{mg} \cdot \mathrm{kg}^{-1}$, 矿化率分别为 $22.2 \% 、 29.7 \%$ 和 $33.3 \%$, 并且不同温度 间都有显著差异 $(p<0.05)$ 。

$Q_{10}$ 是表达土壤有机碳矿化速率与温度之间关 系的较好参数, 多数研究用 $Q_{10}$ 值反映有机碳矿化

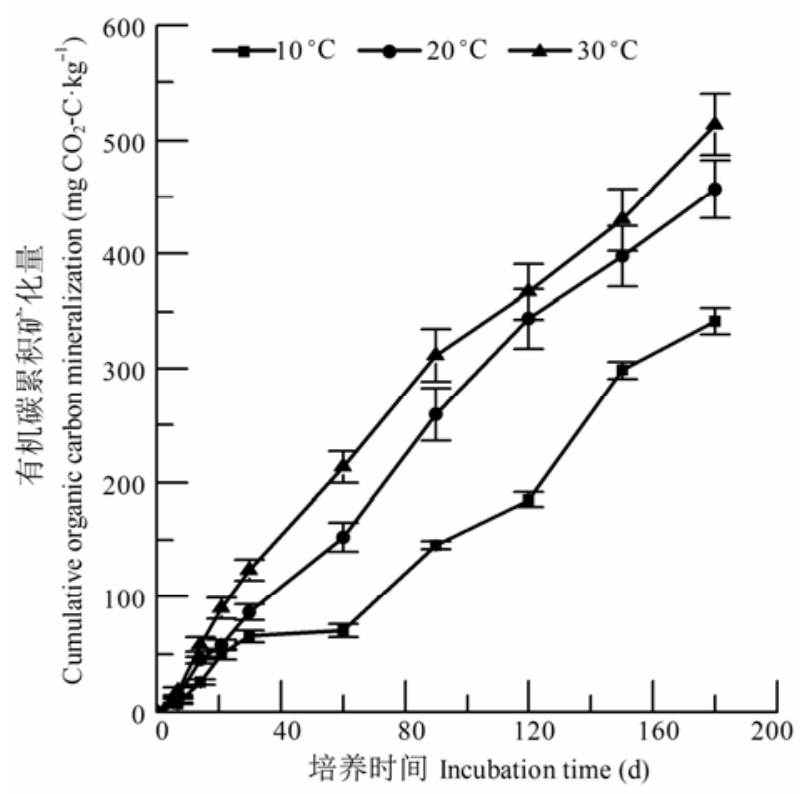

图1 不同温度下严重侵蚀红壤有机碳累积矿化量(平均值 \pm 标准偏差)。

Fig. 1 Cumulative mineralization of soil organic carbon under different temperatures in severely eroded red soil (mean $\pm S D$ ). 
速率对温度的敏感性。严重侵蚀红壤碳矿化在10-20 ${ }^{\circ} \mathrm{C}$ 和 $20-30{ }^{\circ} \mathrm{C}$ 的 $Q_{10}$ 值分别为 $1.41 \pm 0.06$ 和 $1.06 \pm$ 0.10 , 前者显著高于后者 $(p<0.05)$ 。

\section{2 严重侵蚀红壤活性有机碳的变化}

3 个培养温度下, 土壤 $\mathrm{MBC}$ 均随着培养时间增 加而增加(图2A), 且培养30天后土壤MBC随温度增 加呈减少趋势。培养 180 天后, 10、 20 和 $30{ }^{\circ} \mathrm{C}$ 培养的 $\mathrm{MBC}$ 平均值分别为 $19.21 、 17.57$ 和 $16.65 \mathrm{mg} \cdot \mathrm{kg}^{-1}$ 。在 整个培养过程中, 不同温度下土壤DOC含量无显著 性差异 $(p>0.05)$, 但均随着培养时间延长呈减少趋 势 $(p<0.05$, 图2B), 说明温度并没有显著改变土壤 DOC含量, 培养结束时, $10 、 20$ 和 $30{ }^{\circ} \mathrm{C}$ 土壤 $\mathrm{DOC}$ 的 含量分别比初始时 $\left(3.05 \mathrm{mg} \cdot \mathrm{kg}^{-1}\right)$ 降低了 $80.9 \%$ 、 $79.9 \%$ 和 $63.8 \%$ 。

\section{3 严重侵蚀红壤累积矿化量与活性碳的关系}

由图 3 可以看出, 土壤有机碳累积矿化量与 $\mathrm{MBC}$ 显著正相关 $(p<0.05)$, 与 DOC极显著负相关 $(p<0.001)$ 。这表明严重侵蚀红壤的MBC、DOC含 量能够显著影响土壤有机碳的矿化; 在培养过程中 DOC作为碳源被微生物所利用而逐渐减少。

\section{4 严重侵蚀红壤的微生物熵和代谢摘}

土壤微生物熵为 $\mathrm{MBC}$ 含量与土壤有机碳的比 值, 反映土壤微生物对有机碳的利用效率。从图 $4 \mathrm{~A}$ 可见, 3 个温度下微生物熵均随着培养时间显著升高 $(p<0.05)$, 其中第 180 天时 $10 、 20$ 和 $30{ }^{\circ} \mathrm{C}$ 的平均值分 别为 $1.25 \% 、 1.04 \%$ 和 $1.08 \%$, 分别是第 30 天时的 6.05 倍、6.41倍和2.03倍。培养初期微生物熵小, 说明微 生物对土壤有机碳的利用率低; 培养后期较初期显
著升高, 说明随着培养的进行土壤微生物有效提高 了对有机碳的利用率。但第 180 天时不同温度间并没 有显著差异 $(p>0.05)$, 这是由于后期MBC含量差异 不显著导致的。

代谢熵为呼吸速率与 $\mathrm{MBC}$ 含量的比值, 代谢摘 值小, 意味着微生物呼吸消耗碳的比例较小, 建造 微生物细胞碳的比例相对较大，对碳源的利用效率 高。从代谢熵随时间的变化(图4B)可见, 10、20和30 ${ }^{\circ} \mathrm{C}$ 时，代谢熵都随着培养的进行而显著降低 $(p<$ 0.05 ), 其中培养第 180 天时 $10 、 20$ 和 $30{ }^{\circ} \mathrm{C}$ 下的代谢 熵平均值分别为 $0.07 、 0.14$ 和 $0.13 \mathrm{~h}^{-1}$, 分别比培养第 30 天时低 $92.7 \% 、 85.7 \%$ 和 $75.8 \%$; 进一步说明与培 养前期相比，培养后期微生物对土壤碳的利用率提 高了。从温度间的差异可以看出, 培养第 30 天时不 同温度间代谢熵没显著差异 $(p>0.05)$, 但是培养第 90 天和 180 天时 $10{ }^{\circ} \mathrm{C}$ 下的代谢熵显著低于 $20{ }^{\circ} \mathrm{C}$ 和 30 ${ }^{\circ} \mathrm{C}$ 下的代谢熵 $(p<0.05)$ 。

\section{3 讨论}

\section{1 温度对土壤累积矿化量和矿化率的影响}

土壤有机碳矿化是土壤微生物活动的结果, 而 微生物的作用在很大程度上依赖于土壤中养分的供 应。本研究中 $\mathrm{CO}_{2}$ 产生速率呈现出培养前期快、后 期慢的趋势, 与大多数研究结果(Balser \& Wixon, 2009; 王若梦等, 2013; 李杰等, 2014)类似。在培养 前期，土壤中有较多的有机物质为微生物提供养分, 使微生物活动强烈, 土壤有机碳矿化速率很快达到 最高峰，累积矿化量大，随着培养时间的延长，培
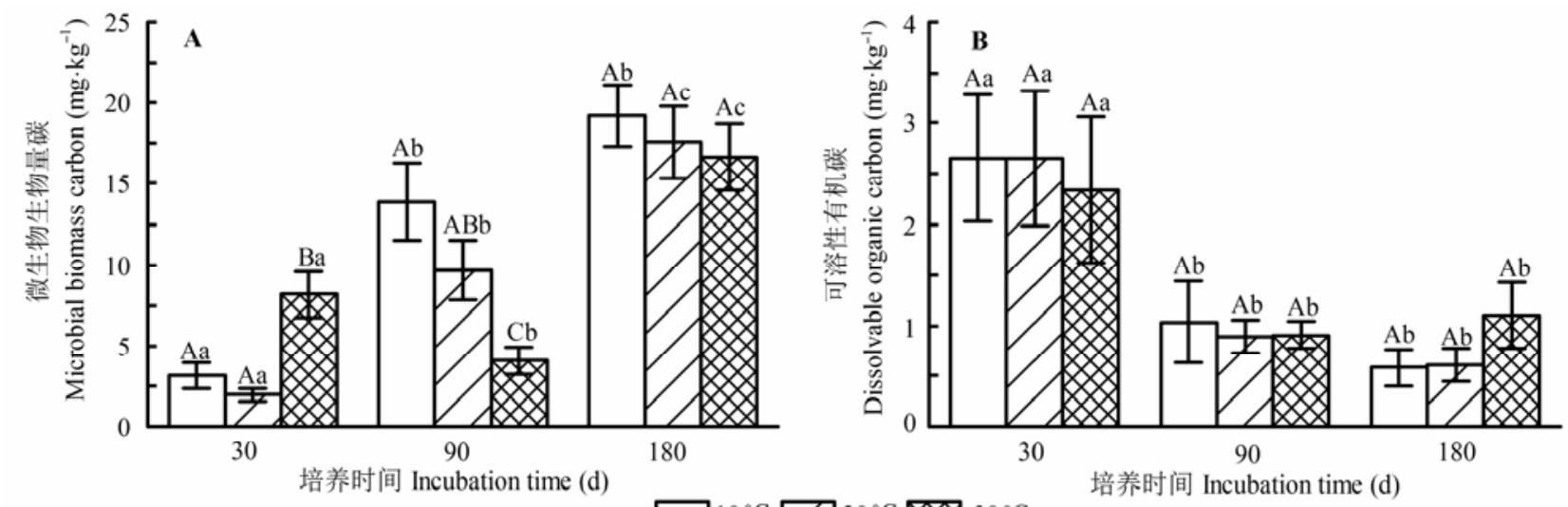

图2 严重侵蚀红壤培养过程中微生物生物量碳和可溶性有机碳的变化(平均值土标准偏差)。不同大写字母表示同一时间不同 温度间差异显著; 不同小写字母表示同一温度不同时间差异显著 $(p<0.05)$ 。

Fig. 2 Change of microbial biomass carbon and dissolved organic matter during incubation period in severely eroded red soil (mean $\pm S D$ ). Different capital letters indicate significant differences between temperature at the same time. Different lowercase letters indicate significant differences between time at the same temperature at $p<0.05$. 

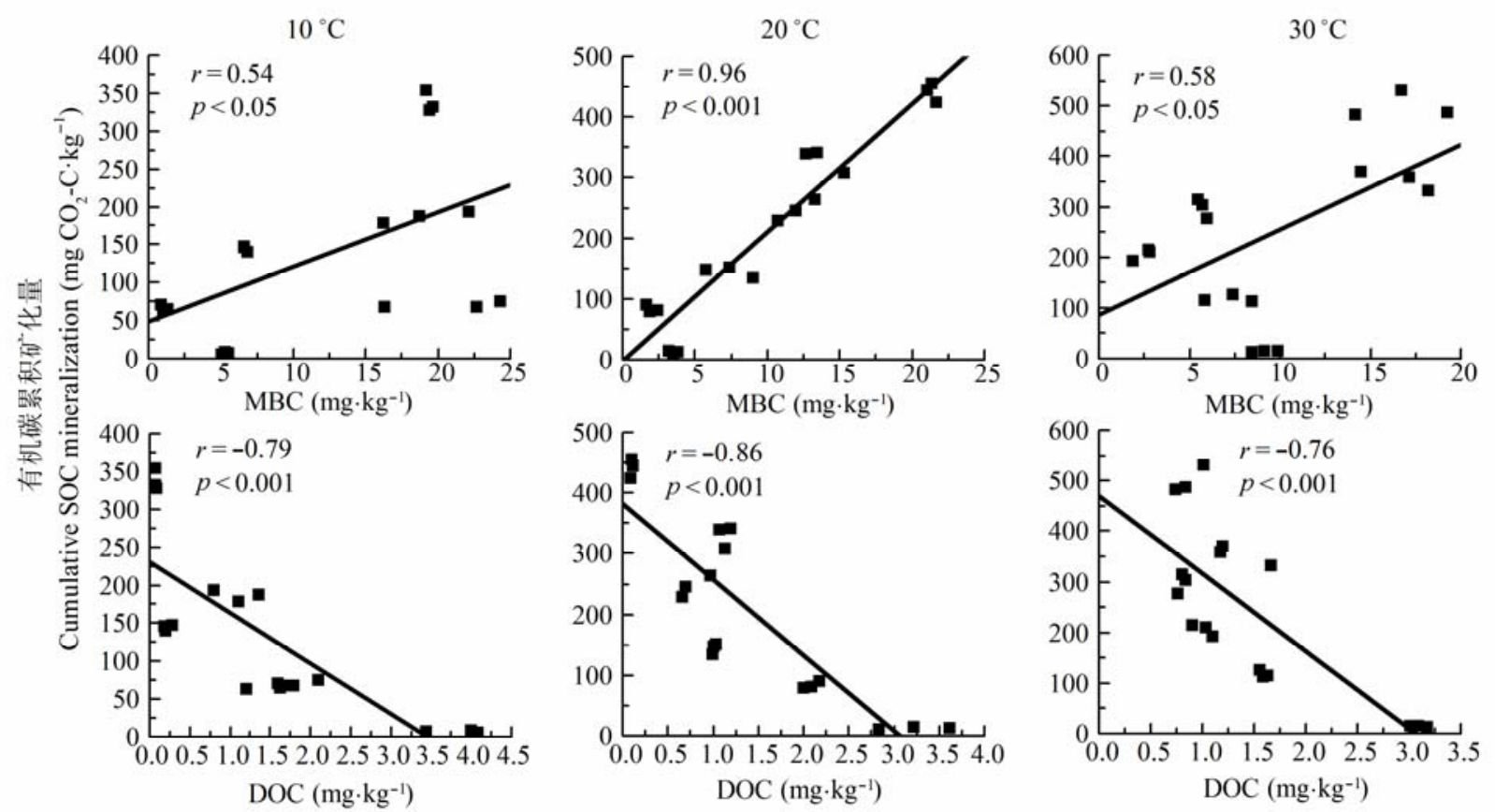

图3＼cjkstart严重侵蚀红壤在不同温度下有机碳累积矿化量与微生物生物量碳(MBC)、可溶性有机碳(DOC)的关系。

Fig. 3 The relationship cumulative soil organic carbon (SOC) mineralization and microbial biomass carbon (MBC) and dissolved organic carbon (DOC) in different incubation temperature of severely eroded red soil.
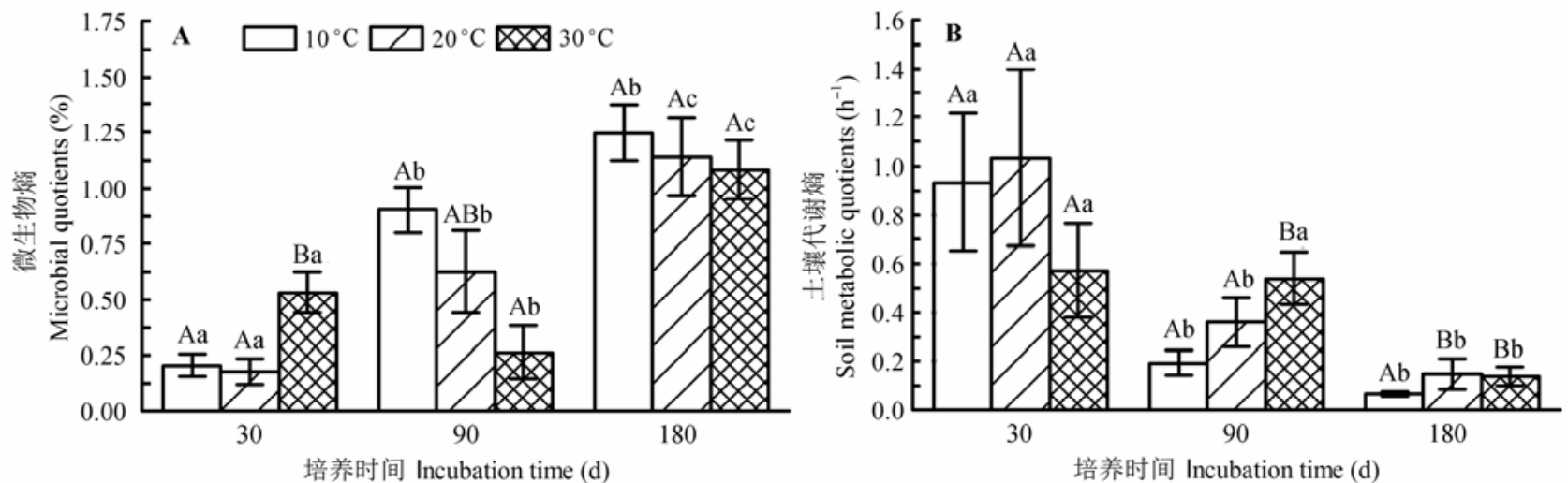

图4 不同温度培养过程中微生物熵和土壤代谢熵随时间的变化(平均值土标准偏差)。不同大写字母表示同一时间不同温度间 差异显著; 不同小写字母表示同一温度不同时间差异显著 $(p<0.05)$ 。

Fig. 4 Change of microbial quotients and soil metabolic quotients in different incubation temperatures during incubation period $($ mean $\pm S D$ ). Different capital letters indicate significant differences between temperature at the same time. Different lowercase letters indicate significant differences between time at the same temperature at $p<0.05$.

养后期土壤中主要为难分解的复杂的纤维素、木质 素等有机碳，微生物活动相对缓和(林杉等, 2014), 累积矿化量小。在培养过程中MBC和DOC与累积矿 化量为显著线性关系(图3), 可溶性有机碳作为碳源 被微生物所利用而逐渐减少(图2B), 这些过程说明 土壤中活性有机碳是有机碳矿化的重要碳源。

温度是影响有机碳矿化的关键因素, 随着温度 的变化, 其他因素的作用也会发生相应的改变。本 研究中经过 180 天的培养, $10 、 20$ 和 $30{ }^{\circ} \mathrm{C}$ 下的有机碳
平均矿化率为 $22.2 \% 、 29.7 \%$ 和 $33.3 \%$, 并且温度间有 显著性差异, 表明随着温度升高土壤有机碳矿化比 例呈递增趋势, 这与大多数研究的结果(王若梦等, 2013; 李杰等, 2014; 林杉等, 2014)一致。然而, 本 研究中侵蚀红壤的有机碳含量很低(表1), 而土壤碳 矿化率与其他研究相比却高得多(表2), 这表明严重 侵蚀红壤有机碳易被分解, 矿化潜力较高。土壤碳 的数量和质量以及微生物对碳的利用效率是影响有 机碳矿化的重要因子。本研究的土壤黏粒含量较低 
表1 土壤基本理化性质(平均值土标准偏差)

Table 1 Physical and chemical characteristics of soil samples (mean $\pm S D)$

\begin{tabular}{|c|c|c|c|c|c|c|c|c|c|c|}
\hline $\begin{array}{l}\text { 实验地 } \\
\text { Sample plot }\end{array}$ & $\begin{array}{c}\text { 有机碳 } \\
\text { Organic C } \\
\left(\mathrm{g} \cdot \mathrm{kg}^{-1}\right)\end{array}$ & $\begin{array}{c}\text { 全氮 } \\
\text { Total } \\
\mathrm{N}\left(\mathrm{g} \cdot \mathrm{kg}^{-1}\right)\end{array}$ & $\begin{array}{c}\text { 可溶性有机碳 } \\
\text { Dissolved organic } \\
\text { carbon } \\
\left(\mathrm{mg} \cdot \mathrm{kg}^{-1}\right)\end{array}$ & $\begin{array}{c}\text { 微生物生物量碳 } \\
\text { Microbial biomass } \\
\text { carbon } \\
\left(\mathrm{mg} \cdot \mathrm{kg}^{-1}\right)\end{array}$ & $\begin{array}{l}\text { 砂粒 } \\
\text { Sand } \\
(\%)\end{array}$ & $\begin{array}{l}\text { 粉粒 } \\
\text { Silt } \\
(\%)\end{array}$ & $\begin{array}{l}\text { 黏粒 } \\
\text { Clay } \\
(\%)\end{array}$ & $\begin{array}{c}\text { 酸碱度 } \\
\mathrm{pH} \text { value }\end{array}$ & $\begin{array}{c}\text { 土壤含水量 } \\
\text { Soil moisture } \\
(\%)\end{array}$ & $\begin{array}{c}\text { 饱和持水量 } \\
\text { Saturation } \\
\text { moisture } \\
\text { capacity }(\%)\end{array}$ \\
\hline $\begin{array}{l}\text { 长汀侵蚀地 } \\
\text { Changting's } \\
\text { eroded land }\end{array}$ & $1.54 \pm 0.06$ & $0.35 \pm 0.03$ & $3.05 \pm 0.22$ & $19.59 \pm 0.96$ & $\begin{array}{c}43.24 \pm \\
3.00\end{array}$ & $\begin{array}{c}37.85 \pm \\
2.00\end{array}$ & $\begin{array}{c}18.91 \pm \\
3.00\end{array}$ & $\begin{array}{c}4.74 \pm \\
0.20\end{array}$ & $\begin{array}{c}15.00 \pm \\
0.25\end{array}$ & $\begin{array}{c}43.00 \pm \\
1.33\end{array}$ \\
\hline
\end{tabular}

表2 不同研究区土壤有机碳矿化率的比较

Table 2 Mineralization rate respired in different study area

\begin{tabular}{|c|c|c|c|c|c|c|}
\hline $\begin{array}{l}\text { 地点 } \\
\text { Site }\end{array}$ & $\begin{array}{l}\text { 土壤类型 } \\
\text { Soil type }\end{array}$ & $\begin{array}{l}\text { 土地利用类型 } \\
\text { Land use type }\end{array}$ & $\begin{array}{l}\text { 培养时间 } \\
\text { Incubation } \\
\text { time (d) }\end{array}$ & $\begin{array}{c}\text { 有机碳含量 } \\
\text { Organic C } \\
\left(\mathrm{g} \cdot \mathrm{kg}^{-1}\right)\end{array}$ & $\begin{array}{c}\text { 矿化率 } \\
\text { Mineralization } \\
\text { rate }(\%)\end{array}$ & $\begin{array}{l}\text { 文献来源 } \\
\text { Literature } \\
\text { resources }\end{array}$ \\
\hline \multirow{7}{*}{$\begin{array}{l}\text { 福建长汀 } \\
\text { Changting, Fujian }\end{array}$} & \multirow[t]{7}{*}{ 红壤 Red soil } & \multirow[t]{7}{*}{ 侵蚀地 Eroded land } & 7 & 1.54 & $0.80\left(20{ }^{\circ} \mathrm{C}\right)$ & \multirow{7}{*}{$\begin{array}{l}\text { 本研究 } \\
\text { This study }\end{array}$} \\
\hline & & & 21 & 1.54 & $3.76\left(20{ }^{\circ} \mathrm{C}\right)$ & \\
\hline & & & 30 & 1.54 & $5.61\left(20{ }^{\circ} \mathrm{C}\right)$ & \\
\hline & & & 60 & 1.54 & $9.87\left(20{ }^{\circ} \mathrm{C}\right)$ & \\
\hline & & & 90 & 1.54 & $16.84\left(20{ }^{\circ} \mathrm{C}\right)$ & \\
\hline & & & 120 & 1.54 & $22.30\left(20{ }^{\circ} \mathrm{C}\right)$ & \\
\hline & & & 180 & 1.54 & $29.68\left(20{ }^{\circ} \mathrm{C}\right)$ & \\
\hline \multirow{2}{*}{$\begin{array}{l}\text { 江苏宜兴 } \\
\text { Yixing, Jiangsu }\end{array}$} & \multirow{2}{*}{$\begin{array}{l}\text { 棕红壤 } \\
\text { Brown-red soil }\end{array}$} & 裸地 Bare land & 20 & 9.23 & $4.12\left(28{ }^{\circ} \mathrm{C}\right)$ & \multirow[t]{2}{*}{ Li et al., 2007} \\
\hline & & $\begin{array}{l}\text { 杉木人工林 } \\
\text { Cunninghamia lanceolata plantation }\end{array}$ & 20 & 9.49 & $2.49\left(28{ }^{\circ} \mathrm{C}\right)$ & \\
\hline \multirow{5}{*}{$\begin{array}{l}\text { 浙江 } \\
\text { Zhejiang }\end{array}$} & \multirow[t]{5}{*}{ 红壤 Red soil } & 裸地 Bare land & 365 & 2.21 & $4.98\left(25{ }^{\circ} \mathrm{C}\right)$ & \multirow{5}{*}{$\begin{array}{l}\text { Xie \& Zhang, } \\
2014\end{array}$} \\
\hline & & 裸地 Bare land & 365 & 4.36 & $2.98\left(25{ }^{\circ} \mathrm{C}\right)$ & \\
\hline & & 裸地 Bare land & 365 & 13.78 & $1.74\left(25{ }^{\circ} \mathrm{C}\right)$ & \\
\hline & & 林地 Forest land & 365 & 29.17 & $3.71\left(25{ }^{\circ} \mathrm{C}\right)$ & \\
\hline & & 林地 Forest land & 365 & 35.32 & $3.91\left(25{ }^{\circ} \mathrm{C}\right)$ & \\
\hline $\begin{array}{l}\text { 福建泉头 } \\
\text { Quantou, Fujian }\end{array}$ & 红壤 Red soil & 果园 Garden land & 85 & 13.21 & $3.91\left(25{ }^{\circ} \mathrm{C}\right)$ & Wang et al., 2012 \\
\hline $\begin{array}{l}\text { 湖南会同 } \\
\text { Huitong, Hunan }\end{array}$ & 红壤 Red soil & $\begin{array}{l}\text { 杉木人工林 } \\
\text { Cunninghamia lanceolata plantation } \\
\text { 常绿阔叶林 } \\
\text { Evergreen broad-leaved forest }\end{array}$ & 21 & 17.00 & $\begin{array}{l}0.09\left(9{ }^{\circ} \mathrm{C}\right) \\
0.12\left(9{ }^{\circ} \mathrm{C}\right)\end{array}$ & Wang et al., 2007 \\
\hline $\begin{array}{l}\text { 福建三明 } \\
\text { Sanming, Fujian }\end{array}$ & 红壤 Red soil & $\begin{array}{l}\text { 杉木人工林 } \\
\text { Cunninghamia lanceolata plantation }\end{array}$ & 28 & 19.23 & $0.92\left(28{ }^{\circ} \mathrm{C}\right)$ & Shen et al., 2012 \\
\hline $\begin{array}{l}\text { 江西吉泰 } \\
\text { Jitai, Jiangxi }\end{array}$ & 红壤 Red soil & $\begin{array}{l}\text { 马尾松林 } \\
\text { Pinus massoniana forest }\end{array}$ & 45 & 9.57 & $1.14\left(25{ }^{\circ} \mathrm{C}\right)$ & Xue et al., 2014 \\
\hline $\begin{array}{l}\text { 福建武夷山 } \\
\text { Mt. Wuyi, Fujian }\end{array}$ & 红壤 Red soil & $\begin{array}{l}\text { 常绿阔叶林 } \\
\text { Evergreen broad-leaved forest }\end{array}$ & 110 & 44.77 & & Zhou et al., 2008 \\
\hline $\begin{array}{l}\text { 江西千烟洲 } \\
\text { Qianyanzhou, } \\
\text { Jiangxi }\end{array}$ & 红壤 Red soil & $\begin{array}{l}\text { 柑橘园 Citrus orchard } \\
\text { 湿地马尾松人工林 } \\
\text { Wetlands Pinus massoniana plantation }\end{array}$ & $\begin{array}{l}7 \\
7\end{array}$ & $\begin{array}{l}11.50 \\
10.12\end{array}$ & $\begin{array}{l}0.05\left(5{ }^{\circ} \mathrm{C}\right) \\
0.06\left(5{ }^{\circ} \mathrm{C}\right)\end{array}$ & Li et al., 2014 \\
\hline
\end{tabular}

括号内为累积矿化率的温度。

Temperature of the cumulative mineralization rate is in brackets.

(表1)。有研究表明, 黏粒含量与土壤有机碳周转时 间关系较密切, 黏粒含量低的土壤有机碳周转快, 有机质易被微生物降解(陈国潮等, 2002)。严重侵蚀 红壤团聚体稳定性低(谢锦升等, 2008), 而团聚体保 护性差的土壤有机碳的矿化率也相对较高(李忠佩 等, 2004; 朱书法等, 2005)。本研究的微生物代谢熵 为 $0.07-1.04 \mathrm{~h}^{-1}$, 明显高于其他研究 (Grego \& Ken- nedy, 1989; 陈璟和杨宁，2013), 如陈璟和杨宁 (2013)对衡阳紫色土丘陵坡地不同恢复阶段土壤的 研究结果表明其微生物代谢熵为 0.039-0.076 $\mathrm{h}^{-1}$, 这是严重侵蚀红壤矿化率高的重要原因。

\section{2 温度对 $Q_{10}$ 的影响}

$Q_{10}$ 表示温度变化对土壤有机碳矿化速率的影 响。许多的土壤室内培养试验结果表明, 在土壤水 
表3 不同研究区土壤温度敏感性 $\left(Q_{10}\right)$ 的比较

Table 3 Temperature sensitivity $\left(Q_{10}\right)$ contrast of soil in different study area

\begin{tabular}{|c|c|c|c|c|c|c|}
\hline $\begin{array}{l}\text { 气候类型 } \\
\text { Climate type }\end{array}$ & $\begin{array}{l}\text { 地点 } \\
\text { Site }\end{array}$ & $\begin{array}{l}\text { 土壤类型 } \\
\text { Soil type }\end{array}$ & $\begin{array}{l}\text { 土地利用类型 } \\
\text { Land use type }\end{array}$ & $\begin{array}{l}\text { 有机碳含量 } \\
\text { Organic C } \\
\left(\mathrm{g} \cdot \mathrm{kg}^{-1}\right)\end{array}$ & $Q_{10}$ & $\begin{array}{l}\text { 文献来源 } \\
\text { Literature resources }\end{array}$ \\
\hline $\begin{array}{l}\text { 亚热带季风气候 } \\
\text { Subtropical monsoon } \\
\text { climate }\end{array}$ & $\begin{array}{l}\text { 福建长汀 } \\
\text { Changting, } \\
\text { Fujian }\end{array}$ & 红壤 Red soil & 侵蚀地 Eroded land & 1.54 & $1.06-1.41$ & $\begin{array}{l}\text { 本研究 } \\
\text { This study }\end{array}$ \\
\hline $\begin{array}{l}\text { 亚热带季风气候 } \\
\text { Subtropical } \\
\text { monsoon climate }\end{array}$ & $\begin{array}{l}\text { 福建泉头 } \\
\text { Quantou, Fujian }\end{array}$ & 红壤 Red soil & 果园 Garden land & 13.21 & 1.49 & Wang et al., 2012 \\
\hline 亚热带季风气候 & 江西千烟洲 & 红壤 Red soil & 柑橘园 Citrus orchard & 11.50 & 3.40 & Li et al., 2014 \\
\hline $\begin{array}{l}\text { Subtropical } \\
\text { monsoon climate }\end{array}$ & Qianyanzhou, Jiangxi & & $\begin{array}{l}\text { 湿地马尾松人工林 } \\
\text { Wetlands Pinus massoniana } \\
\text { plantation }\end{array}$ & 10.12 & 2.20 & \\
\hline $\begin{array}{l}\text { 亚热带季风气候 } \\
\text { Subtropical } \\
\text { monsoon climate }\end{array}$ & $\begin{array}{l}\text { 广东鼎湖 } \\
\text { Dinghu, Guangdong }\end{array}$ & $\begin{array}{l}\text { 赤红壤 Lateritic } \\
\text { red soil }\end{array}$ & $\begin{array}{l}\text { 针阔叶混交林 Mixed } \\
\text { coniferous and broad-leaved } \\
\text { forest }\end{array}$ & 26.80 & 2.03 & Deng et al., 2007 \\
\hline $\begin{array}{l}\text { 亚热带季风气候 } \\
\text { Subtropical }\end{array}$ & $\begin{array}{l}\text { 福建南平 } \\
\text { Nanping, Fujian }\end{array}$ & 红壤 Red soil & $\begin{array}{l}\text { 杉木人工林 Cunninghamia } \\
\text { lanceolata plantation }\end{array}$ & 22.60 & 1.40 & Wei \& Ma, 2006 \\
\hline $\begin{array}{l}\text { monsoon } \\
\text { climate }\end{array}$ & & & $\begin{array}{l}\text { 马尾松人工林 Pinus } \\
\text { massoniana } \text { plantation }\end{array}$ & 20.90 & 1.39 & \\
\hline $\begin{array}{l}\text { 亚热带季风气候 } \\
\text { Subtropical } \\
\text { monsoon climate }\end{array}$ & $\begin{array}{l}\text { 四川盆地 } \\
\text { Sichuan Basin }\end{array}$ & 红壤 Red soil & $\begin{array}{l}\text { 林地 Forestland } \\
\text { 裸地 Bare land }\end{array}$ & $\begin{array}{l}19.26 \\
11.98\end{array}$ & $\begin{array}{l}1.25-3.25 \\
1.54-3.68\end{array}$ & Wang et al., 2007 \\
\hline $\begin{array}{l}\text { 亚热带季风气候 } \\
\text { Subtropical } \\
\text { monsoon climate }\end{array}$ & $\begin{array}{l}\text { 湖南 } \\
\text { Hunan }\end{array}$ & 水稻土 Paddy soil & $\begin{array}{l}\text { 水稻田 } \\
\text { Paddy field }\end{array}$ & 19.20 & $2.11-2.78$ & Ren et al., 2007 \\
\hline $\begin{array}{l}\text { 干燥亚湿润气候 } \\
\text { Dry sub-humid climate }\end{array}$ & $\begin{array}{l}\text { 内蒙古科尔沁沙地 } \\
\text { Horqin sandy lands, Nei } \\
\text { Mongol }\end{array}$ & $\begin{array}{l}\text { 风沙土 } \\
\text { Aeolian } \\
\text { sandy soil }\end{array}$ & $\begin{array}{l}\text { 裸地 } \\
\text { Bare land }\end{array}$ & 3.36 & $1.55-1.97$ & Wang et al., 2008 \\
\hline $\begin{array}{l}\text { 高原气候 } \\
\text { Plateau climate }\end{array}$ & $\begin{array}{l}\text { 青藏高原 } \\
\text { Qinghai-Xizang Plateau }\end{array}$ & $\begin{array}{l}\text { 草甸土 } \\
\text { Meadow soil }\end{array}$ & $\begin{array}{l}\text { 封育草地 } \\
\text { Enclosure grassland } \\
\text { 自由放牧草地 } \\
\text { Grazing grassland }\end{array}$ & $\begin{array}{l}19.04 \\
10.30\end{array}$ & $\begin{array}{l}1.46-1.99 \\
1.41-2.11\end{array}$ & Xu et al., 2013 \\
\hline $\begin{array}{l}\text { 温带季风气候 } \\
\text { Temperate monsoon } \\
\text { climate }\end{array}$ & $\begin{array}{l}\text { 吉林长白山 } \\
\text { Changbai Mountain, } \\
\text { Jilin }\end{array}$ & $\begin{array}{l}\text { 草甸土 } \\
\text { Meadow soil }\end{array}$ & $\begin{array}{l}\text { 原生林 Original forest } \\
\text { 次生林 Secondary forest }\end{array}$ & $\begin{array}{l}8.06 \\
4.03\end{array}$ & $\begin{array}{l}1.23-1.61 \\
1.23-1.61\end{array}$ & Wang et al., 2013 \\
\hline
\end{tabular}

分不受限制的情况下，土壤碳矿化的温度敏感性随 着温度的增加而下降。本研究中 $10-20{ }^{\circ} \mathrm{C}$ 和 $20-30{ }^{\circ} \mathrm{C}$ 下的 $Q_{10}$ 值分别为 1.41 和 $1.06, Q_{10}$ 值随温度增加而下 降，与前人的多数研究结果一致。Niklińska等(1999) 沿欧洲大陆气候样带取7个欧洲赤松(Pinus sylvestris)林下土壤腐殖质样品，在持水量(WHC)为 $50 \%$ 、 5-25 ${ }^{\circ} \mathrm{C}$ 下进行14周室内培养, 发现样带北部土壤在 $10-15{ }^{\circ} \mathrm{C}$ 时, 土壤呼吸的 $Q_{10}$ 超过 5 , 但在 $25{ }^{\circ} \mathrm{C}$ 附近 时, $Q_{10}$ 约为 1 。Leifeld和Fuhrer (2005)对采自农田和 草地的土壤样品在不同温度下连续培养发现, 在 15-25 ${ }^{\circ} \mathrm{C}$ 时, $Q_{10}$ 值为 5.2 ; 而在 $25-30{ }^{\circ} \mathrm{C}$ 时, $Q_{10}$ 值为 2.8 。土壤微生物作为有机碳矿化的动力, 是决定 $Q_{10}$ 的内在因子, 同时对温度变化的响应十分敏感。本 研究中, 随着培养时间的增加, 微生物商逐渐升高, 表明微生物对有机碳的同化效率逐渐提高, 这可能 是微生物群落中真菌逐渐增加而细菌逐渐减少的缘 故。Zhang等(2005)的研究表明：真菌比细菌的同化
效率更高, 土壤中真菌数量增加, 而且温度升高也 会提高真菌的生物量, 促进惰性有机碳组分的分 解。土壤微生物对碳的利用效率的变化也能影响有 机碳矿化(Reichstein et al., 2000)。本研究中，培养90 天后，土壤微生物的代谢熵随着温度升高而增加， 这说明一定时间后有机碳矿化对温度的响应更敏 感; 因为温度升高会抑制不适应高温的细菌生长, 而促进真菌生长，提高真菌对惰性组分的分解利用， 这一结果亦证实了我们上述的推测, 也就是说, 培 养初期细菌利用的土壤有机碳的活性组分 (Zogg et al., 1997)随着培养的进行逐渐减少(图2B); 然而微 生物生物量是增加的, 这可能是由真菌生物量的提 高引起的。Zhang等(2005)对北美高草草原人工升温 $2{ }^{\circ} \mathrm{C}$ 土壤进行磷脂脂肪酸分析发现: 升温使真菌优 势度增加, 细菌优势度降低, 微生物群落结构发生 转变, 降低了土壤异养呼吸 $Q_{10}$ 。因此, 微生物群落 组成和对碳利用效率的改变可能是 $Q_{10}$ 随温度升高 
而降低的主要原因。

周涛等(2009)的研究表明: 中国由北到南的黑 棕壤、棕壤、褐土、黄壤、红壤、赤红壤和砖红壤 的 $Q_{10}$ 值分别为 $1.61 、 1.54 、 1.63 、 2.1 、 1.87 、 2.21$ 和 1.78 。而本研究的 $Q_{10}$ 值为 1.41 和 1.06 , 低于大多数 研究结果(表3), 特别是 $20-30{ }^{\circ} \mathrm{C}$ 时的 $Q_{10}$ 值接近于 1 。呼吸底物有效性是决定 $Q_{10}$ 的重要因子(同小娟等, $2005)$, 研究表明呼吸底物质量越高, $Q_{10}$ 值越高 (Melillo et al., 2002; Fang et al., 2005)。Macdonald等 (1999)对美国密歇根州硬木林灰土的培养研究发现: 底物质量较高的表层土壤呼吸的 $Q_{10}$ 高于底物质量 较低的底层土壤呼吸的 $Q_{10}$ 。然而, 土壤微生物对温 度的适应性对 $Q_{10}$ 具有重要影响, 本研究中的严重 侵蚀红壤长期处于裸露状态, 温度变幅大, 如夏季 地表温度高达 $40{ }^{\circ} \mathrm{C}$ 以上, 土壤微生物对高温的适应 可能降低土壤碳矿化的温度敏感性。Balser和Wixon (2009)的研究表明土壤微生物群落的适应能力与土 壤有机质的质量和呼吸底物有效性无关。Lange和 Green (2005)的研究表明真菌的呼吸对土壤温度的 季节性变化有明显的适应性, 如果土壤微生物对高 温环境产生适应性, 那么在较高温度时会出现较低 的 $Q_{10}$ (Luo et al., 2001)。因此, 土壤微生物的适应性 也是严重侵蚀红壤 $Q_{10}$ 值在较高温度时显著低于较 低温度时的一个原因。

基金项目国家重点基础研究发展规划(“973”计 划) (2012CB722203和2014CB954003)和国家自然科 学基金(31370465和31130012)。

致谢 感谢福建师范大学地理科学学院的任卫岭、 陈云玉、蒲晓婷、刘志江、张秋芳、周嘉聪、郑永 和游章活等同学在实验中给予的帮助。

\section{参考文献}

Ai L, Wu JG, Zhu G, Liu JQ, Tian ZQ, Chang W, Xia X (2007). The mineralization of alpine meadow soil organic carbon and factors influencing it in the Qilian Mountain. Acta Prataculturae Sinica, 16(5), 22-33. (in Chinese with English abstract) [艾丽, 吴建国, 朱高, 刘建泉, 田自强, 茬伟, 夏新 (2007). 祁连山中部高山草甸土壤有机碳矿 化及其影响因素研究. 草业学报, 16(5), 22-33.]

Balser TC, Wixon DL (2009). Investigating biological control over soil carbon temperature sensitivity. Global Change Biology, 12, 2935-2949.

Chatterjee A, Vance GF, Pendall E, Stahl PD (2008). Timber harvesting alters soil carbon mineralization and microbial community structure in coniferous forests. Soil Biology \& Biochemistry, 40, 1901-1907.

Chen GC, He ZL, Huang CY (2002). Turnover of microbial biomass $\mathrm{C}$ in red soils and its significance in soil fertility evaluation. Acta Pedologica Sinica, 39, 152-160. (in Chinese with English abstract) [陈国潮, 何振立, 黄昌勇 (2002). 红壤微生物生物量 C周转及其研究. 土壤学报, 39, 152-160.]

Chen J, Yang N (2013). Changes in SBR and $q \mathrm{CO}_{2}$ at different re-vegetation stages on sloping land with purple soils in Hengyang of Hunan Province, south-central China. Journal of Tropical and Subtropical Botany, 6, 514-520. (in Chinese with English abstract) [陈璟, 杨宁 (2013). 衡阳 紫色土丘陵坡地不同恢复阶段土壤基础呼吸及代谢熵 的变化. 热带亚热带植物学报, 6, 514-520.]

Conant RT, Drijber RA, Haddix ML, Parton WJ, Paul EA, Plante AF (2008). Sensitivity of organic matter decomposition to warming varies with its quality. Global Change Biology, 4, 868-877.

Deng Q, Liu SZ, Liu JX, Meng Z, Zhang DQ (2007). Contributions of litter-fall to soil respiration and its affacting factors in southern subtropical forests of China. Advances in Earth Science, 9, 976-986. (in Chinese with English abstract) [邓琦, 刘世忠, 刘菊秀, 孟泽, 张德强 (2007). 南亚热带森林凋落物对土壤呼吸的贡献及其影响因素. 地球科学进展, 9, 976-986.]

Fang CM, Pete S, Moncrieff JB, Smith JU (2005). Similar response of labile and resistant soil organic matter pools to changes in temperature. Nature, 433, 57-59.

Grego S, Kennedy AY (1989). Effect of ammonitrate and stabilized farm yard manure on microbial biomass and metabolic quotient of soil under Zea mays. Agriculture of Mediterranean, 128, $132-137$.

Hashimoto S, Tanaka N, Suzuki M, Inoue A, Takizawa H, Kosaka I, Tanaka K, Tantasirin C, Tangtham N (2004). Soil respiration and soil $\mathrm{CO}_{2}$ concentration in a tropical forest, Thailand. Journal of Forest Research, 9, 75-79.

He SJ, Xie JS, Yang ZJ, Yin YF, Li DC, Yang YS (2011). Status, causes and prevention of soil and water loss in Pinus massoniana woodland in hilly red soil region of southern. China Science of Soil and Water Conservation, 9(6), 65-70. (in Chinese with English abstract) [何圣嘉, 谢锦升, 杨智杰, 尹云锋, 李德成, 杨玉盛 (2011). 南 方红壤丘陵区马尾松林下水土流失现状、成因及防治. 中国水土保持科学, 9(6), 65-70.]

IPCC (Intergovernmental Panel on Climate Change) (2013). Contribution of working group 1 to the Fifth Assessment Report of the intergovernmental panel on climate change. In: Allen SK, Plattner GK, Nauels A, Xia Y, Stocker TF eds. Climate Change in 2013: The Physical Science Basis. Cambridge University Press, Cambridge, UK. 
Jenkinson D, Adams D, Wild A (1991). Model estimates of $\mathrm{CO}_{2}$ emissions from soil in response to global warming. Nature, 351, 304-306.

Lal R (2004). Soil carbon sequestration impacts on global climate change and food security. Science, 304, 1623-1627.

Lange OL, Green TGA (2005). Lichens show that fungi can acclimate their respiration to seasonal changes in temperature. Oecologia, 1, 11-19.

Leifeld J, Fuhrer J (2005). The temperature response of $\mathrm{CO}_{2}$ production from bulk soils and soil fractions is related to soil organic matter quality. Biogeochemistry, 75, 433-453.

Li J, Wei XH, Chai H, Wang RM, Wang D, He NP (2014). Impacts of land-use types on soil $\mathrm{C}$ mineralization and temperature sensitivity of forests in Qianyanzhou, Jiangxi Province, China. Chinese Journal of Applied Ecology, 7, 1919-1926. (in Chinese with English abstract) [李杰, 魏 学红, 柴华, 王若梦, 王丹, 何念鹏 (2014). 土地利用 类型对千烟洲森林土壤碳矿化及其温度敏感性的影响. 应用生态学报, 7, 1919-1926.]

Li ZG (2009). Current condition and dynamic changes of soil erosion in China. China Water Resources, 12, 8-11. (in Chinese with English abstract) [李智广 (2009). 中国水 土流失现状与动态变化. 中国水利, 12, 8-11.]

Li ZP, Wu XC, Chen BY (2007). Changes in transformation of soil organic carbon and functional diversity of soil microbial community under different land use patterns. Scientia Agricultura Sinica, 8, 1712-1721. (in Chinese with English abstract) [李忠佩, 吴晓晨, 陈碧云 (2007). 不同利 用方式下土壤有机碳转化及微生物群落功能多样性变 化. 中国农业科学, 8, 1712-1721.]

Li ZP, Zhang TL, Chen BY (2004). Dynamics of soluble organic carbon and its relation to mineralization of soil organic carbon. Acta Pedologica Sinica, 41, 544-552. (in Chinese with English abstract) [李忠佩, 张桃林, 陈碧云 (2004). 可溶性有机碳的含量动态及其与土壤有机碳矿 化的关系. 土壤学报, 41, 544-552.]

Lin S, Chen T, Zhao JS, Xiang RB, Hu RG, Zhang SQ, Wang ML, Lü ZQ (2014). Characteristics of soil organic carbon mineralization at different temperatures in paddy soils under long-term fertilization. Chinese Journal of Applied Ecology, 5, 1340-1348. (in Chinese with English abstract) [林杉, 陈涛, 赵劲松, 向荣彪, 胡荣桂, 张水清, 王米 兰, 吕昭琪 (2014). 不同培养温度下长期施肥水稻土的 有机碳矿化特征. 应用生态学报, 5, 1340-1348.]

Liu SH, Fang JY (1997). Effect factors of soil respiration and the temperature's effects on soil respiration in the global scale. Acta Ecologica Sinica, 17, 469-476. (in Chinese with English abstract) [刘绍辉, 方精云 (1997). 土壤呼 吸的影响因素及全球尺度下温度的影响. 生态学报, 17, 469-476.]

Lu RK (1999). Methods of Soil Agricultural Chemical Analysis.
Chinese Agricultural Science and Technology Press, Beijing. (in Chinese) [鲁如坤 (1999). 土壤农业化学分析方 法. 中国农业科技出版社, 北京.]

Luo Y, Wan S, Hui D, Wallace LL (2001). Acclimatization of soil respiration to warming in a tall grass prairie. Nature, 413, 622-625.

Macdonald NW, Randlett DL, Zak DR (1999). Soil warming and carbon loss from a lake states spodosol. Soil Science Society of America Journal, 63, 211-218.

Melillo JM, Steudler PA, Aber JD, Newkirk K, Lux H, Bowles FP (2002). Soil warming and carbon-cycle feedbacks to the climate system. Science, 298, $2173-2176$.

Niklińska M, Maryański M, Laskowski R (1999). Effect of temperature on humus respiration rate and nitrogen mineralization: Implications for global climate change. Biogeochemistry, 3, 239-257.

Reichstein M, Bednorz F, Broll G, Kätterer T (2000). Temperature dependence of carbon mineralization: Conclusions from a long-term incubation of subalpine soil samples . Soil Biology \& Biochemistry, 7, 947-958.

Ren XE, Tong CL, Sun ZL, Tang GY, Xiao HA, Wu JS (2007). Effects of temperature on organic carbon mineralization in paddy soils with different clay content. Chinese Journal of Applied Ecology, 10, 2245-2250. (in Chinese with English abstract) [任秀娥, 童成立, 孙中林, 唐国勇, 肖和艾, 吴金水 (2007). 温度对不同黏粒含量稻田土壤有机碳 矿化的影响. 应用生态学报, 10, 2245-2250.]

Shen FF, Yuan YH, Fan HB, Liu WF, Liu YQ (2012). Effects of elevated nitrogen deposition on soil organic carbon mineralization and soil enzyme activities in a Chinese fir plantation. Acta Ecologica Sinica, 32, 517-527. (in Chinese with English abstract) [沈芳芳, 袁颖红, 㚞后保, 刘 文飞, 刘苑秋 (2012). 氮沉降对杉木人工林土壤有机碳 矿化和土壤酶活性的影响. 生态学报, 32, 517-527.]

Tong XJ, Tao B, Cao MK (2005). The responses of soil respiration and nitrogen mineralization to global warming in terrestrial ecosystems. Progress in Geography, 4, 84-96. (in Chinese with English abstract) [同小娟, 陶波, 曹明奎 (2005). 陆地生态系统土壤呼吸、氮矿化对气候变暖的 响应. 地理科学进展, 4, 84-96.]

Wang D, Lü YL, Xu L, He X, Xu ZW, Zhao N, Wang RL, He NP (2013). Impact of changes in vegetation types on soil C mineralization and associated temperature sensitivity in the Changbai Mountain forests of China. Acta Ecologica Sinica, 19, 6373-6381. (in Chinese with English abstract) [王丹, 吕瑜良, 徐丽, 何秀, 徐志伟, 赵宁, 王瑞丽, 何 念鹏 (2013). 植被类型变化对长白山森林土壤碳矿化 及其温度敏感性的影响. 生态学报, 19, 6373-6381.]

Wang F, Wang YX, Chen YZ, Wu ZD, Jiang FY, You ZM, Zhang WJ, Weng BQ (2012). Effects of temperature and green manure treatments on organic carbon mineralization 
in orchard soil. Journal of Southern Agriculture, 12, 1991-1996. (in Chinese with English abstract) [王峰, 王 义祥, 陈玉真, 吴志丹, 江福英, 尤志明, 张文锦, 翁伯 琦 (2012). 不同温度及化肥绿肥施用比例对果园土壤 有机碳矿化的影响. 南方农业学报, 12, 1991-1996.]

Wang H, Fan ZP, Deng DZ, Chen DL, Sun XK, Gao JG, Zeng DH (2008). Effects of environmental factors on soil organic carbon mineralization in a Pinus sylvestris var. mongolica plantation. Chinese Journal of Ecology, 9, 1469-1475. (in Chinese with English abstract) [王红, 范 志平, 邓东周, 陈德龙, 孙学凯, 高俊刚, 曾德慧 (2008). 不同环境因子对樟子松人工林土壤有机碳矿化 的影响. 生态学杂志, 9, 1469-1475.]

Wang QK, Wang SL, Yu XJ, Zhang J, Liu YX (2007). Soil carbon mineralization potential and its effect on soil active organic carbon in evergreen broad-leaved forest and Chinese fir plantation. Chinese Journal of Ecology, 12, 1918-1923. (in Chinese with English abstract) [王清奎, 汪思龙, 于小军, 张剑, 刘燕新 (2007). 常绿阔叶林与 杉木林的土壤碳矿化潜力及其对土壤活性有机碳的影 响. 生态学杂志, 12, 1918-1923.]

Wang RM, Dong KH, He NP, Zhu JX, Dai JZ, Shi KK (2013). Effect of enclosure on soil $\mathrm{C}$ mineralization and priming effect in Stipa grandis grassland of Inner Mongolia. Acta Ecologica Sinica, 12, 3622-3629. (in Chinese with English abstract) [王若梦, 董宽虎, 何念鹏, 朱剑兴, 代景 忠, 施㑆㑆 (2013). 围封对内蒙古大针茅草地土壤碳矿 化及其激发效应的影响. 生态学报, 12, 3622-3629.]

Wang XG, Zhu B, Wang YQ, Zheng XH (2007). Soil respiration and its sensitivity to temperature under different land use conditions. Acta Ecologica Sinica, 5, 1960-1968. (in Chinese with English abstract) [王小国, 朱波, 王艳强, 郑循华 (2007). 不同土地利用方式下土壤呼吸及其温 度敏感性. 生态学报, 5, 1960-1968.]

Wei HD, Ma XQ (2006). Dynamics of soil respiration in three major plantations in mid-subtropical zone. Journal of Fujian Agriculture and Forestry University (Natural Science Edition), 3, 272-277. (in Chinese with English abstract) [尉海东, 马祥庆 (2006). 中亚热带3 种主要人工林的土 壤呼吸动态. 福建农林大学学报 (自然科学版), 3, 272-277.]

Xie GX, Zhang MK (2014). Influence of biochar application on mineralization and fractions of organic carbon in red soils with different land use. Chinese Journal of Soil Science, 2, 413-419. (in Chinese with English abstract) [谢国雄, 章 明奎 (2014). 施用生物质炭对红壤有机碳矿化及其组 分的影响. 土壤通报, 2, 413-419.]

Xie JS, Yang YS, Chen GS, Zhu JM, Zeng HD, Yang ZJ (2008). Effects of vegetation restoration on water stability and organic carbon distribution in aggregates of degraded red soil in subtropics of China. Acta Ecologica Sinica, 2, 702-709. (in Chinese with English abstract) [谢锦升, 杨 玉盛，陈光水，朱锦禁，曾宏达，杨智杰 (2008). 植被 恢复对退化红壤团聚体稳定性及碳分布的影响. 生态 学报, 2, 702-709.]

Xu Li, Yu SX, He NP, Wen XF, Shi PL, Zhang YJ, Dai JZ, Wang RM (2013). Soil C mineralization and temperature sensitivity in alpine grasslands of the Qinghai-Xizang Plateau. Chinese Journal of Plant Ecology, 11, 988-997. (in Chinese with English abstract) [徐丽, 于书霞, 何念鹏, 温学发, 石培礼, 张扬建, 代景忠, 王若梦 (2013). 青 藏高原高寒草地土壤碳矿化及其温度敏感性. 植物生 态学报, 11, 988-997.]

Xue JY, Zhang HX, Quan Q, Wang RM, Gan YM, He NP (2014). Effect of land-use type on soil carbon mineralization and its priming effect on red soils in the mid-subtropics of China. Chinese Journal of Applied and Environmental Biology, 3, 516-522. (in Chinese with English abstract) [薛晶 月, 张洪轩, 全权, 王若梦, 干友民, 何念鹏 (2014). 土 地利用方式对中亚热带红壤碳矿化及其激发效应的影 响. 应用与环境生物学报, 3, 516-522.]

Zhang W, Parker KM, Luo Y, Wan S, Wallace LL, Hu S (2005). Soil microbial responses to experimental warming and clipping in a tall grass prairie. Global Change Biology, 11, 266-277.

Zhou T, Shi PJ, Hui DF, Luo YQ (2009). The temperature sensitivity of soil respiration spatial pattern of inversion in China. Scientia Sinica Vitae, 3, 315-322. (in Chinese) [周 涛, 史培军, 惠大丰, 骆亦其 (2009). 中国土壤呼吸温 度敏感性空间格局的反演. 中国科学 $\mathrm{C}$ 辑: 生命科学, 3 , 315-322.]

Zhou Y, Xu XG, Ruan HH, Wang JS, Fang YH, Wu YY, Xu ZK (2008). Mineralization rates of soil organic carbon along an elevation gradient in Wuyi Mountain of Southeast China. Chinese Journal of Ecology, 27, 1901-1907. (in Chinese with English abstract) [周炎, 徐宪根, 阮宏 华, 汪家社, 方燕鸿, 吴焰玉, 徐自坤 (2008). 武夷山 不同海拔高度土壤有机碳矿化速率的比较. 生态学杂 志, 27, 1901-1907.]

Zhu SF, Liu CQ, Tao FX (2005). Use of $\delta^{13} \mathrm{C}$ method in studying soil organic matter. Acta Pedologica Sinica, 42, 495-503. (in Chinese with English abstract) [朱书法, 刘 从强, 陶发祥 (2005). $\delta^{13} \mathrm{C}$ 方法在土壤有机质研究中的 应用. 土壤学报, 42, 495-503.]

Zogg GP, Zak DR, Ringelberg DB, MacDonald NW, Pregitzer KS, White DC (1997). Compositional and functional shifts in microbial communities due to soil warming. Soil Science Society of America Journal, 61, 475-481.

责任编委: 程晓莉 责任编辑: 王 藏 


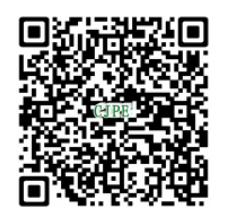

植物生态学报官网

唯一投稿网址

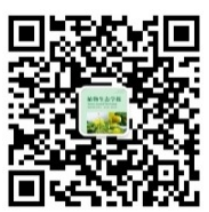

微信订阅号

期刊及学科

相关信息发布

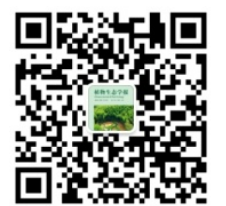

微信服务号

稿件状态查询

全文检索浏览 